\title{
The Difference between Developmental Dyslexia and Dysgraphia: Recent Neurobiological Evidence
}

\author{
Filippos Vlachos*, Elias Avramidis \\ Department of Special Education, University of Thessaly, Greece \\ Received December 17, 2019; Revised February 25, 2020; Accepted March 12,2020
}

Copyright@2020 by authors, all rights reserved. Authors agree that this article remains permanently open access under the terms of the Creative Commons Attribution License 4.0 International License

\begin{abstract}
Developmental dyslexia and developmental dysgraphia are considered to be distinct learning difficulties that affect the child's ability to learn. Dyslexia affects all aspects of written language, while the symptoms of dysgraphia appear to be confined to difficulties in writing. However, the distinction between the two learning difficulties is often obscured by the similar learning cognitive deficits manifested by individuals diagnosed with these difficulties. The aim of this review is to summarize and evaluate research concerning the neurobiological basis of the two difficulties with a view to assist researchers and practitioners in their classification of individuals with resembling deficits. In so doing, we bring together the findings of studies that have utilized powerful neuroimaging techniques such as functional Magnetic Resonance Imaging (fMRI). The evidence suggests that while individuals with dyslexia and dysgraphia share a left-hemisphere processing limitation resulted from the absence of a scanning mechanism for disembedding, encoding, and rehearsing visual patterns (e.g., words), there are important neurological differences that distinguish the two groups. Specifically, recent neurobiological studies have shown that children with developmental dysgraphia differ from their dyslexic counterparts in white matter integrity, functional connectivity revealed by fMRI, and white matter-gray matter correlations. These differences in the brain between children with developmental dyslexia and children with dysgraphia confirm the neurobiological distinction between these two special learning difficulties. Based on these neurobiological differences researchers and practitioners in the field should exercise special care not to treat the two disorders as the same in their research or professional practice.
\end{abstract}

Keywords Developmental Dyslexia, Developmental Dysgraphia, Neurobiological Differences

\section{Introduction}

Developmental dyslexia and developmental dysgraphia are closely related developmental disorders that can exist together as well as separately [1]. Although the literature contains different classifications and definitions for these two disorders, they are often used interchangeably by practitioners in the field.

Developmental dyslexia is the most common learning disability in school-aged children. It is genetic in origin with a neurobiological substrate. It is characterized by a marked impairment in the development of reading skills and affects a considerable number of children [2-4]. Although difficulties in accurate and/or fluent word reading are at the core of the definition of developmental dyslexia, the latter is not just a reading disorder [3, 5]. It is also a writing disorder because of the spelling mistakes that emerge with the production of written texts; nevertheless, the writing problems in developmental dyslexia remain under-recognised and under-treated [6]. Young children identified as at risk for being diagnosed with the disorder may also experience delayed language development and have trouble learning to spell and write as they reach school age. That is why in the case of a child struggling with reading or writing, it can be difficult to pinpoint exactly what the problem is.

Developmental dysgraphia may be defined as a failure in the normal development of writing skills [7]. Writing skills are below those expected for a person's age or ability, despite appropriate education. It may contribute significantly to a child's learning difficulty or learning disability and is a matter of both educational and medical significance [8]. Some children diagnosed with developmental dysgraphia have developed strong verbal skills to compensate for their writing issues and are often strong readers. Because little is known about the disorder, it often remains unidentified by educators and other professionals in the field. This could be partly attributed to the significant handwriting and spelling problems 
exhibited in dysgraphic learners which often lead practitioners to confuse developmental dysgraphia with the more commonly found developmental dyslexia, despite the dysgraphic learners' unimpaired reading skills.

The Diagnostic and Statistical Manual of Mental Disorders (4th edition) distinguishes between three categories of learning disabilities, namely: reading disability, mathematical disability and 'disorder of written expression', together with a fourth category, 'learning disability not otherwise specified'. Developmental dysgraphia as a disorder of written expression is characterised by "writing skills (that) ....are substantially below those expected given the person's chronological age, measured intelligence, and age-appropriate education" [9]. However, in this definition there is a lack of clarity as to whether writing refers only to the motor skill of writing (which is the traditional interpretation) or whether it also includes the orthographic skill of spelling [10].

In the 5th edition of the Diagnostic and Statistical Manual of Mental Disorders (DSM-V), developmental dysgraphia no longer exists as a separate category but falls under the category of "specific learning disorder." This category refers to difficulties in learning and to limited academic skills, as indicated by the presence of at least one of the following symptoms that have persisted for at least 6 months despite the provision of interventions that target those difficulties: inaccurate or slow and effortful word reading; or difficulty understanding the meaning of what is read, or with spelling, or with written expression, or mastering number sense, number facts, or calculation; or difficulties with mathematical reasoning [11].

In sum, both developmental dyslexia and developmental dysgraphia affect the child's ability to learn. It must be stressed, however, that although they are considered to be two distinct learning difficulties that affect the child's learning ability in a very different way, little is known about their neurological differences. The aim of this review is to summarize and evaluate recent empirical research, which supports the distinction between developmental dyslexia and dysgraphia not only at the behavioral but also at the neurobiological level.

\section{Similarities and Differences}

Dyslexia affects all aspects of written language, while the symptoms of dysgraphia appear to be confined to difficulties in handwriting [1]. For this reason, in the process of diagnosing dysgraphia and in order to give a student the corresponding characterization, it is also necessary that reading ability and spelling should not be deficient. In a recent review, Döhla and Heim concluded that both learning disabilities have diverse comorbidities and predictors within and outside the language domain in common. Examining how far existing knowledge about the cognitive causes of developmental dyslexia also apply to developmental dysgraphia, Döhla and Heim suggest that phonological awareness plays an important role in both dyslexia and dysgraphia. Additionally, they assume that further predictors such as working memory, auditory processing, visual attention, and language impairment play an important role in reading and writing as well [1].

Another recent review synthesized neuroimaging studies carried out in adults and in children with and without learning disabilities with a view to identifying the neural network involved in handwriting and reading. This review concluded that the mastery of handwriting is based on the involvement of a network of brain structures which is built upon the joint learning of writing and reading. A part of this graphomotor network is brought into play during the identification of letters through visual reading. Additionally, both writing and reading form the basis for the accomplishment of more complex language tasks involving orthographic knowledge such as the composition of texts [12].

In another review, Nicolson and Fawcett sought to identify the similarities and differences between developmental dyslexia and dysgraphia. They concluded that both of these learning disabilities reflect lack of automaticity at the cognitive level, which can be attributed to impaired circuits of procedural learning located at the cerebellum. Accordingly, the authors suggest that 'pure' dysgraphia reflects an impairment of the cerebellar-motor circuit and 'pure' dyslexia reflects an impairment of the cerebellar-language circuit [10].

In regards to the neurobiological cause of developmental dyslexia and dysgraphia, in a previous published study, Mather proposed the concurrent tapping/line orientation procedure. This procedure aimed to investigate brain-processing differences in early adolescence with dysgraphia, dyslexia and good readers/spellers. This study demonstrated that individuals with dyslexia and dysgraphia are different from healthy individuals, having a print-scanning deficit in common. From this study the author concluded that individuals with dyslexia and dysgraphia share a left-hemisphere processing limitation resulted from the absence of a scanning mechanism for disembedding, encoding, and rehearsing visual patterns (e.g., words). This limitation could have arisen due to introducing written language prematurely to children late in developing left-hemisphere motor dominance [13].

Apart from the above mentioned findings in children, another study examined the cognitive mechanisms and neural substrates of phonological dyslexia and dysgraphia, in a large cohort of adult patients with focal damage to perisylvian cortical regions that had affected phonological processing. Lesion-deficit correlations indicated that phonological dyslexia and dysgraphia may be produced by a damage in a variety of perisylvian cortical regions, consistent with distributed network models of phonological processing. Their results provide empirical 
support for shared-components models of written language processing, according to which the same central cognitive systems support both reading and spelling. [14].

Overall, the above mentioned studies indicate that writing and reading share common brain and cognitive networks. Such findings suggest that developmental dyslexia and dysgraphia might have a similar neurobiological basis.

\section{Recent Neurobiological Evidence}

Many neuroimaging studies indicate that the disruption of left hemisphere posterior brain systems underlies the symptoms of developmental dyslexia [15-17]. Such findings are in line with the suggestion of the greater right hemisphere involvement in the expression of developmental dyslexia revealed by behavioral studies [18].

As regards dysgraphia, a review of the neural correlates of dysgraphia derived from lesion studies and studies using neuroimaging and assessment of minor neurological dysfunction concluded that our knowledge on the neural substrate of paediatric dysgraphia is limited. The limited available data suggest that at least some of the children with developmental dysgraphia have dysfunctions in extensive supraspinal networks, involving cortical areas in all regions of the brain, while in others, dysfunction may be restricted to either the cerebellum or specific cortical sites [19].

However, the advent of more sophisticated neuroimaging technologies over the last years, allowed brain activation to be measured objectively. Recent neuroimaging studies provide evidence on the distinction between these two specific learning difficulties at the neurobiological level.

In a recent study, researchers using fMRI (functional Magnetic Resonance Imaging: a magnetic resonance imaging-based technique that makes it possible to detect the brain areas that are involved in a task) and DTI (Diffusion Tensor Imaging: a neuroimaging technique which makes it possible to estimate the location, orientation, and anisotropy of the brain's white matter tracts) examined three groups of children (dyslexics, dysgraphics and typical developers) aged 9-15 years. Having administered graphic tasks, they concluded that the three groups differed from each other in graphic and cognitive tasks. The control group had more interfaces to the white matter, which facilitate functional links to the gray matter for language and cognitive work. Conversely, children with developmental dyslexia and developmental dysgraphia showed less white matter connections and more functional links to gray matter sites - in other words, their brains had to work harder to achieve the same tasks. As the researchers note, their brains were less effective in language processing. Additionally, the results showed that students with dysgraphia and students with dyslexia differed in white matter integrity, fMRI functional connectivity, and white matter-gray matter correlations. Such findings provide evidence that the two specific learning difficulties are not the same, because functional links were not the same in children with dyslexia and those with dysgraphia, either in writing or in cognitive tasks [20].

Another study examined whether students in grades 4 to 9 with persistent problems with written language beyond the first three school grades could be classified into one of the three Specific Learning Disability (SLD) diagnostic categories defined on the basis of level of language impairment: developmental dysgraphia (impaired subword handwriting), developmental dyslexia (impaired word spelling and reading), or oral and written language learning disability (impaired oral and written syntax comprehension and expression). In this study, language impairment was assessed through administering normed tests and parent questionnaires as well as examining the students' recorded developmental and educational histories. Furthermore, this study examined whether these three diagnostic groups might also have different neuroimaging profiles in relation to fMRI functional connectivity in brain locations associated with processing and producing written words on a word-specific spelling task to other brain locations. The findings confirmed unique diagnostic patterns based on impairments in levels of language. Moreover, these results provided converging fMRI functional connectivity brain evidence that those with developmental dysgraphia, developmental dyslexia, oral and written language learning disability, and controls differ in their neuroimaging profiles. Additionally, the three SLD groups differed in the number of functional connections made from the same local brain region to other brain regions during a common word-specific spelling task. Based on behavioral and brain evidence, researchers concluded that developmental dysgraphia, developmental dyslexia, and oral and written language learning disability appear to be discreet SLDs, even if they share some commonalities [21].

In sum, both the aforementioned studies provide evidence that the brain differences observed in developmental dysgraphia and developmental dyslexia on written language tasks are consistent with their behavioral impairments in handwriting and/or in word spelling.

\section{Discussion \& Conclusions}

There is a considerable overlap between dyslexia and dysgraphia since they are both developmental language disorders that can manifest themselves in similar ways. For example, in both disorders converting phonemes (sounds) into graphemes (letters in written form) constitute major 
challenges for children. Additionally, developmental dyslexia and developmental dysgraphia are disorders that can occur by themselves or in conjunction with other difficulties thus rendering the line between them more blurred. However, despite the similarities that can be observed in the problems experienced by children diagnosed with dyslexia and dysgraphia, recent explorations of their underlying cause at the neural systems level have revealed significant differences between the two disorders. Specifically, recent neurobiological studies have shown for the first time that children with dysgraphia differ from their dyslexic counterparts in white matter integrity, fMRI functional connectivity, and white matter-gray matter correlations [20; 21]. These significant brain differences between children with dyslexia and children with dysgraphia confirm the neurobiological distinction between these two special learning difficulties. In this respect, researchers and practitioners in the field should exercise special care not to treat the two disorders as the same in their research or professional practice.

A further observation that can be made from the available literature concerns the existence of distinct subtypes of developmental dysgraphia. For example, research has identified three subtypes of developmental dysgraphia, namely 'dyslexic dysgraphia' that bears similarities to dyslexia; dysgraphia due to motor clumsiness; and dysgraphia due to defect in understanding of space [22]. Each subtype places the emphasis on different deficits underlying the difficulties experienced by children with dysgraphia including linguistic learning, working memory, and motor planning [23]. In a similar vein, a recent study of German 3rd graders with developmental dysgraphia detected the existence of two subtypes of dysgraphia, namely phonological and surface. While both subgroups showed subnormal skills in visual-graphemic tasks, the phonological dysgraphic children showed poorer performance in different phonological tasks than the children assigned to the surface subtype [24]. Notwithstanding the value of such distinctions, a caveat needs pointing out; these distinctions emanate largely from behavioral manifestations instead of solid neurobiological evidence. Future research efforts could, therefore, focus on examining the neurobiological bases of different types of developmental dysgraphia resulting in firm classifications of children diagnosed with this disorder. In doing so, effective interventions can be designed which are tailored to meet the needs of children with different types of developmental dysgraphia.

\section{REFERENCES}

[1] Döhla D, Heim S, Developmental Dyslexia and Dysgraphia: What can We Learn from the One about the other? Frontiers in Psychology. 2016; 6: 2045.
[2] Shastry B, Developmental dyslexia: An update. Journal of Human Genetics. 2007; 52: 104-109.

[3] Vlachos F, Dyslexia: A synthetic approach to causal theories. Hellenic Journal of Psychology. 2010; 7: 205-240.

[4] Vlachos F, Avramidis E, Dedousis G, Chalmpe M, Ntalla I, Giannakopoulou, M, Prevalence and gender ratio of dyslexia in Greek adolescents and its association with parental history and brain injury. American Journal of Educational Research. 2013; 1: 22-25.

[5] Fletcher J, Dyslexia: The evolution of a scientific concept. Journal of the International Neuropsychological Society. 2009; 15: 501-508.

[6] Berninger VW, Winn W, Stock P, Abbott RD, Eschen K, Lin SJ, et al. Tier 3 specialized writing instruction for students with dyslexia. Reading \& Writing, 2008; 21: 95-129.

[7] Vlachos F, Karapetsas A, Visual memory deficits in children with dysgraphia. Perceptual \& Motor Skills.2003; 97: 1281-1288.

[8] Gubbay S, de Klerk N, A study and review of developmental dysgraphia in relation to acquired dysgraphia. Brain \& Development. 1995; 17: 1-8.

[9] American Psychiatric Association. Diagnostic and Statistical Manual of Mental Disorders, 4th edition (DSM-IV). Washington, DC: American Psychiatric Association. 2000.

[10] Nicolson R, Fawcett A, Dyslexia, dysgraphia, procedural learning and the cerebellum. Cortex. 2011; 47: 117-127.

[11] American Psychiatric Association. Diagnostic and Statistical Manual of Mental Disorders, 5th edition (DSM-V). Arlington, VA: American Psychiatric Publishing. 2013.

[12] Longcamp M, Richards, TL, Velay J-L, \& Berninger VW, Neuroanatomy of Handwriting and Related Reading and Writing Skills in Adults and Children with and without Learning Disabilities: French-American Connections. Pratiques. 2016; 171-172: 3175.

[13] Mather D, Dyslexia and Dysgraphia: More Than Written Language Difficulties in Common. Journal of Learning Disabilities. 2003; 36: 307-317.

[14] Rapcsak SZ, Beeson PM, Henry ML, Leyden A, Kim E, Rising $\mathrm{K}$, et al. Phonological Dyslexia and Dysgraphia: Cognitive Mechanisms and Neural Substrates. Cortex. 2009; 45: 575-591.

[15] Shaywitz B, Shaywitz S, Pugh K, Mencl W, Fulbright R, Skudlarski P, et al. Disruption of posterior brain systems for reading in children with developmental dyslexia. Biological Psychiatry. 2002; 52: 101-110.

[16] Eckert MA, Berninger VW, Vaden KI, Gebregziabher M, Tsu L, Gray Matter Features of Reading Disability: A Combined Meta-Analytic and Direct Analysis Approach. eNeuro, 2016; 3(1): ENEURO.0103-15.2015.

[17] Mascheretti S, De Luca A, Trezzi V, Peruzzo D, Nordio A, Marino C, et al. Neurogenetics of developmental dyslexia: from genes to behavior through brain neuroimaging and cognitive and sensorial mechanisms. Translational Psychiatry. 2017; 7(1): e987.

[18] Vlachos F, Andreou E, Delliou A,. Brain hemisphericity and 
developmental dyslexia. Research in Developmental Disabilities. 2013; 34: 1536-1540.

[19] Van Hoorn JF, Maathuis CGB, Hadders-Algra M. Neural correlates of paediatric dysgraphia. Dev Med Child Neurol. 2013; 55:65-68.

[20] Richards TL, Grabowski TJ, Boord P, Yagle K, Askren M, Mestre Z, et al. Contrasting brain patterns of writing-related DTI parameters, fMRI connectivity, and DTI-fMRI connectivity correlations in children with and without dysgraphia or dyslexia. NeuroImage: Clinical. 2015; 8: 408-421.

[21] Berninger VW, Richards T, Abbott RD, Differential Diagnosis of Dysgraphia, Dyslexia, and OWL LD: Behavioral and Neuroimaging Evidence. Reading and Writing. 2015; 28(8); 1119-1153.

[22] Deuel RK, Developmental dysgraphia and motor skills disorders. Journal of Child Neurology. 1995; 10(1): S6-S8.

[23] McCloskey M, Rapp B, Developmental dysgraphia: An overview and framework for research, Cognitive Neuropsychology. 2017; 34(3-4): 65-82.

[24] Cholewa, J. Mantey, S Heber, S., Hollweg, W. Developmental surface and phonological dysgraphia in German 3rd graders. Reading and Writing. 2010; 23(1): 97-127. 